\title{
Highly compact refractive index sensor based on stripe waveguides for lab-on-a-chip sensing applications
}

\author{
Chamanei Perera ${ }^{1}$, Kristy Vernon ${ }^{* 1}$, Elliot Cheng ${ }^{2}$, Juna Sathian ${ }^{3}$, Esa Jaatinen ${ }^{1}$ \\ and Timothy Davis ${ }^{4}$
}

\author{
Full Research Paper \\ Address: \\ ${ }^{1}$ Science and Engineering Faculty, Queensland University of \\ Technology, Brisbane 4001, Queensland, Australia, ${ }^{2}$ Australian \\ National Nanofabrication Facility QLD node, University of \\ Queensland, St Lucia 4072, Queensland, Australia, ${ }^{3}$ Department of \\ Materials, Imperial College, London SW7 2AZ, UK and ${ }^{4}$ School of \\ Physics, University of Melbourne, Parkville 3010, Victoria, Australia

\section{Email:} \\ Kristy Vernon* - k.vernon@qut.edu.au \\ * Corresponding author

\section{Keywords:} \\ interferometer; sensing; surface plasmons; waveguides \\ Beilstein J. Nanotechnol. 2016, 7, 751-757. \\ doi:10.3762/bjnano.7.66 \\ Received: 11 February 2016 \\ Accepted: 12 May 2016 \\ Published: 25 May 2016 \\ Associate Editor: C. Jagadish \\ (c) 2016 Perera et al; licensee Beilstein-Institut. \\ License and terms: see end of document.
}

\begin{abstract}
In this paper we report the design and experimental realisation of a novel refractive index sensor based on coupling between three nanoscale stripe waveguides. The sensor is highly compact and designed to operate at a single wavelength. We demonstrate that the sensor exhibits linear response with a resolution of $6 \times 10^{-4}$ RIU (refractive index unit) for a change in relative output intensity of $1 \%$. Authors expect that the outcome of this paper will prove beneficial in highly compact, label-free and highly sensitive refractive index analysis.
\end{abstract}

\section{Introduction}

Plasmons are coherent oscillations of free electrons existing on metal dielectric interfaces and are highly sensitive to the surrounding dielectric environment. This unique property is incredibly useful in sensing applications. Mach-Zehnder (MZ) interferometry [1-5], surface enhanced Raman spectroscopy (SERS) [6-9], ring resonators [10] and surface plasmon resonance (SPR) [11-13] are widely used techniques utilising plasmons to measure refractive index changes.

SPR is the most widely used sensing technique. It provides high sensitivity with label-free detection. Most commercially avail- able SPR sensors employ a prism configuration to excite SPs on a metal surface which makes the device too large for lab-on-achip applications [3] and requires precise alignments [12,14].

Nano-plasmonic sensors utilising metallic nanostructures can be used to overcome these limitations. In addition, they allow miniaturisation of the overall device size as well as improving the ease of excitation. The plasmonic Mach-Zehnder interferometer (MZI) is one such alternative passive nano-optical device used in refractive index sensing applications $[3,5,15,16]$. In physics, a MZI is a device used to determine the relative 
phase shift variations between two collimated beams derived from splitting light from the same single source [17]. These nano-sized MZI based sensors typically have a sensitivity 10-100 times less than that of the commercially available SPRprism based sensors, but have the advantage of being more compact and having sufficient sensitivity for being used in some lab-on-a-chip applications [3].

Batoli et al. have designed a MZI integrated on a microfluidic platform consisting of two parallel nanoslits in a metal film coated on a glass substrate $[3,18]$. One slit was used to scatter white light into SP modes on a metal/fluidic interface (sample) on top and a metal/glass interface (reference) at the bottom. Launched SPs on the top and bottom surfaces travelled along the interfaces toward the other slit. These two SP modes interfered with each other and modulated the far-field scattering at this slit. Far-field intensity depends on the phase shift between two SP modes. The resolution of this MZI was $1.5 \times 10^{-4}$ RIU while the minimum size of the device had a lateral dimension of $23 \mu \mathrm{m}$.

Pacifici at el. also developed an MZI consisting of two grooves separated by a slit [5]. White light was directed onto the grooves and excited multiple SPs with different frequencies. Counter propagating SP waves interfered with the incident light at the slit location causing modification of the light intensity transmitting through the slit. The intensity of the transmitted light carries information about the near-field interaction of the plasmon with the dielectric environment. The resolution of this sensor was $3 \times 10^{-7}$ RIU.

All of the interferometric designs described require exciting a SP on a metal slab with a surface defect (such as a groove or slit) and then analysing the resulting interference pattern transmitted through a slit. These sensors enable excitation using white light beams and generate interference patterns from multifrequency SPs. Most of the presented MZI designs are at least micron sized and it is difficult to analyse two analytes at once. As an example, the sensor proposed by Pacifi et al. needs to run separately for reference and sample solutions to allow analysis [5].

MZIs can be designed to be wavelength specific and more compact using waveguide structures. Vernon et al. proposed a compact interferometer design using stripe waveguide coupling to measure the change in the refractive index of a sample using the change in the output intensity [19]. The stripe waveguides in the proposed design were supporting long-range surface plasmon polaritons (LRSPPs). The design consisted of four stripes in total. Light coupled into the sensor using the input arm with the generated LRSPP travelling along the input arm before coupling into the two outer reference and sample arms. Waves propagating along the two outer arms then evanescently coupled into the output arm. The presence of a sample in the sample arm caused a phase change in the wave travelling in the sample arm. This phase shift was then detected by the change in the output intensity at the output arm.

In this paper, we report the realisation of a refractive index sensor based on stripe waveguide coupling (see Figure 1). The structure is similar to the structure proposed by Vernon et al. [19] but without the output coupling arm, thus reducing the overall size of the device. The sample window was etched on top of the sample arm via a second electron beam lithography (EBL) process, and was used to place sample solution on top of the sample arm. The presence of the sample changes the wavenumber of the propagating plasmon mode hence changes the output intensity at the end of the sample arm. The sample arm and reference arms must be separated from the input arm sufficiently so that the propagating modes do not couple between the sample and reference arm, only between the input arm and the outer arms. The length of the waveguides must also be chosen to ensure there is full transfer of energy between the input and outer arms (i.e., the lengths of the arms are determined by the coupling length of the system). A schematic of the interferometer design is given in Figure 1.

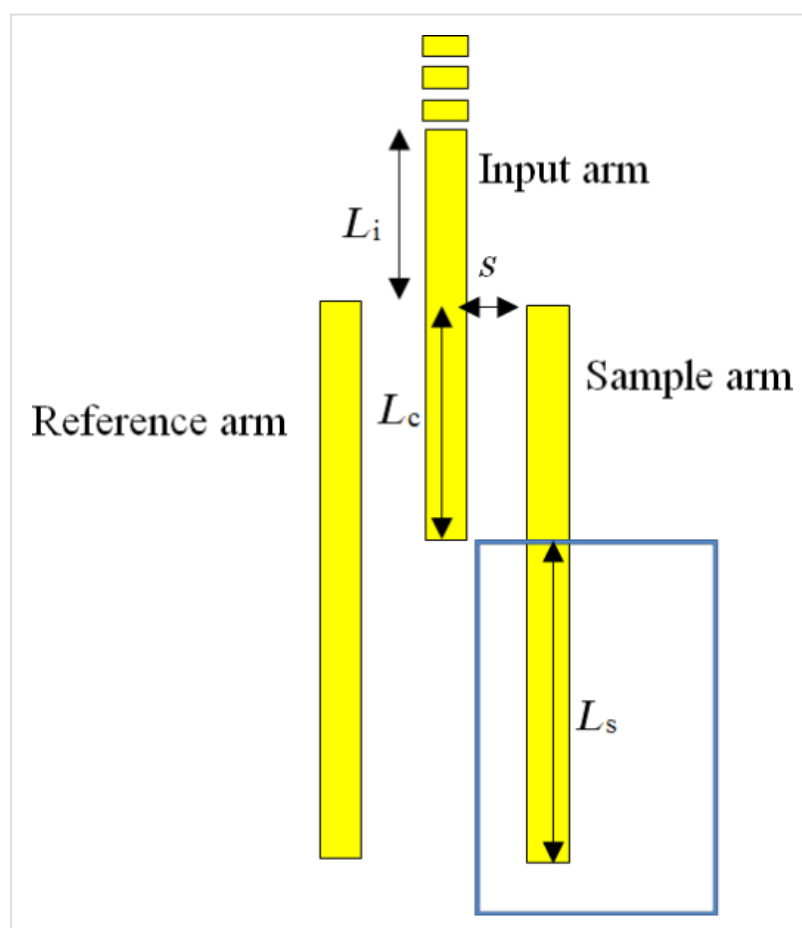

Figure 1: Schematic diagram of the proposed sensor. The sample window is shown as the blue rectangle. $L_{\mathrm{i}}$ is the length of the input waveguide before coupling with the outer arms, $L_{c}$ is the coupling length, $L_{s}$ is the sample length and $s$ is the separation between inner arm and outer arms. 


\section{Results and Discussion}

\section{Theory}

For the MZI based on stripe waveguides, the waveguide is required to support a single LRSPP mode $\left(\mathrm{ss}_{\mathrm{b}}{ }^{0}\right)$ at $633 \mathrm{~nm}$ excitation wavelength. Previously it has been reported [20] that this can be achieved with a silver stripe waveguide of width $750 \mathrm{~nm}$ and thickness $30 \mathrm{~nm}$. The overall sensor design consists of three identical stripe waveguides labelled as input arm, reference arm and sample arm (Figure 1). The $\mathrm{ss}_{\mathrm{b}}{ }^{0}$ mode of the input arm can be excited via end-fire excitation or grating coupling. This mode propagates along the input arm until its evanescent field starts to interact with the two outer arms.

$L_{\mathrm{i}}$ is the length of the input waveguide before coupling with the outer arms, $L_{\mathrm{c}}$ is the coupling length and $L_{\mathrm{S}}$ is the sample length. The sample arm and reference arm are separated from the input arm by a separation distance $s$, and are uncoupled to each other. This situation can produce only three eigenmodes as shown in Figure 2. For more detail on these eigenmodes and COMSOL simulations refer to [19].

The total field of the coupled system is a linear combination of these three eigenmodes and can be derived using the Haus and Fonstad approach [21]. Refer to Supporting Information File 1 for more details. The parameters of the proposed design are sep- aration distance $(s) 200 \mathrm{~nm}, L_{\mathrm{i}}=5 \mu \mathrm{m}, L_{\mathrm{c}}=29 \mu \mathrm{m}$ and $L_{\mathrm{s}}=20 \mu \mathrm{m}$.

\section{Intensity analysis}

We are interested in the output intensity difference at the sample arm and the reference arm. The corresponding $z$ at this point is

$$
z=L_{\mathrm{i}}+L_{\mathrm{c}}+L_{\mathrm{s}}
$$

The electric field of the reference arm at this point is

$$
E_{\mathrm{r}}=a_{\mathrm{r}} e^{i k_{\mathrm{r}}\left(z-L_{\mathrm{i}}-L_{\mathrm{c}}\right)}
$$

where $k_{\mathrm{r}}$ is the wavenumber of the mode travelling along the reference arm. The intensity from the reference arm (i.e., at $z=L_{\mathrm{i}}+L_{\mathrm{c}}+L_{\mathrm{s}}$ ) can be determined using

$$
I_{\mathrm{r}}=\left|E_{\mathrm{r}}\right|^{2}
$$

The wavenumber of the propagating mode on the stripe waveguide changes due to the presence of a sample. The electric field of the sample arm at $z=L_{\mathrm{i}}+L_{\mathrm{c}}+L_{\mathrm{S}}$ is given by
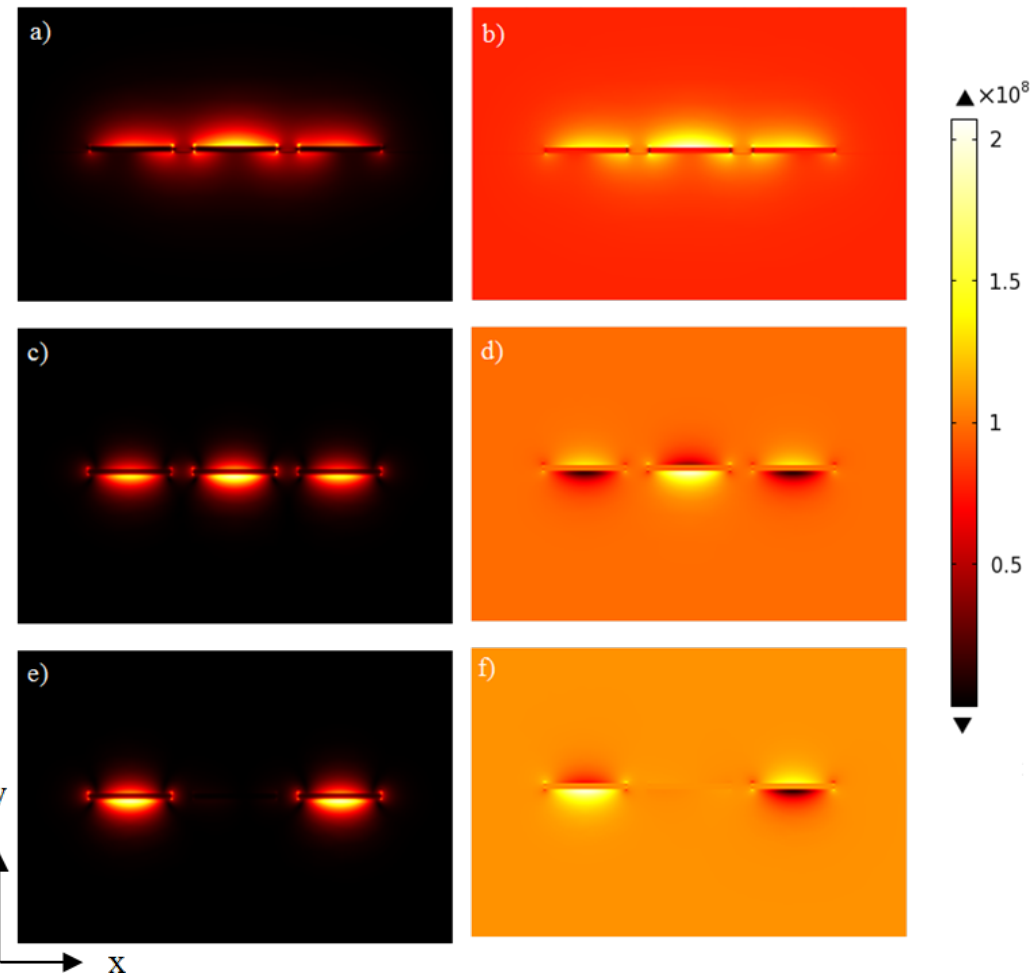

Figure 2: (a), (c) and (e): Magnitude of the electric field distribution of the eigenmodes 1, 2 and 3. (b), (d) and (f): The $y$-component of the electric field for eigenmodes 1, 2 and 3. Stripes are made from silver, $750 \mathrm{~nm}$ wide, $30 \mathrm{~nm}$ thick, and separated by $200 \mathrm{~nm}$ on ITO coated glass substrate with PMMA cladding for an excitation wavelength of $633 \mathrm{~nm}$. These simulations were done using COMSOL Multiphysics. 


$$
E_{\mathrm{s}}=a_{\mathrm{s}} e^{i k_{\mathrm{s}}\left(z-L_{\mathrm{i}}-L_{\mathrm{c}}\right)}
$$

and the corresponding intensity can be found using

$$
I_{\mathrm{s}}=\left|E_{\mathrm{s}}\right|^{2} \text {. }
$$

To determine the sensitivity, we analyse the intensity difference between reference arm and sample arm due to a small change in the sample refractive index.

\section{Fabrication}

The refractive index sensor (RI sensor) with a sample window on top of the sample arm was fabricated using a multi-step e-beam lithography (EBL) technique. First, the RI sensor designs and alignment marks were patterned on a $300 \mathrm{~nm}$ bilayer PMMA resist (950 k A4 / 495 k A4 PMMA resist from Microchem $\mathrm{GmbH}$ ) using electron beam lithography (JEOL7800 FE-SEM with Raith Quantum Elphy) with a beam current of $\approx 75 \mathrm{pA}$ and $20 \mathrm{kV}$ acceleration voltage under optimal dose of $280 \mu \mathrm{C} / \mathrm{cm}^{2}$. The patterned PMMA bilayer was then developed for 30 seconds in MIBK/IPA 1:3 solution. $30 \mathrm{~nm} \mathrm{Ag}$ was evaporated on the developed sample using the PVD 75 e-beam evaporator under a slow rate of 0.1 angstrom per second. A scanning electron microscope image was obtained (Figure 3) which shows the successfully survived sensor designs after lift-off in acetone bath. Second, a $300 \mathrm{~nm}$ bilayer PMMA resist was spincoated on top of the structures to pattern the sample window on top of the sample arm. Then, the sample windows were patterned in the second EBL step. Finally, the patterned bilayer was developed in the developer solution for 30 seconds.

Lengths of the input guide, reference arm and sample arm were $15 \mu \mathrm{m}, 25 \mu \mathrm{m}$ and $25 \mu \mathrm{m}$ respectively. The design was tested for two separation distances of $200 \mathrm{~nm}$ and $300 \mathrm{~nm}$ as shown in Figure 3.

\section{Testing}

A grating structure was placed at the input end to increase the energy transfer of incoming light into the input waveguide [22] The gratings were excited through an inverted microscope setup using a $633 \mathrm{~nm}$ wavelength laser, further details are provided in [22]. The plasmon propagation and coupling in the RI sensor was imaged using CdSe quantum dots (QDs) with emission wavelength at $655 \mathrm{~nm}$ (from Invitrogen Cat. No. Q21321MP). An optimum QD spacer layer thickness of $18 \mathrm{~nm} \mathrm{SiO} 2$ was selected [23]. The sensor designs with separations between two outer arms of $300 \mathrm{~nm}$ (Figure 4a) and $200 \mathrm{~nm}$ (Figure 4b) were observed under QD luminescence.

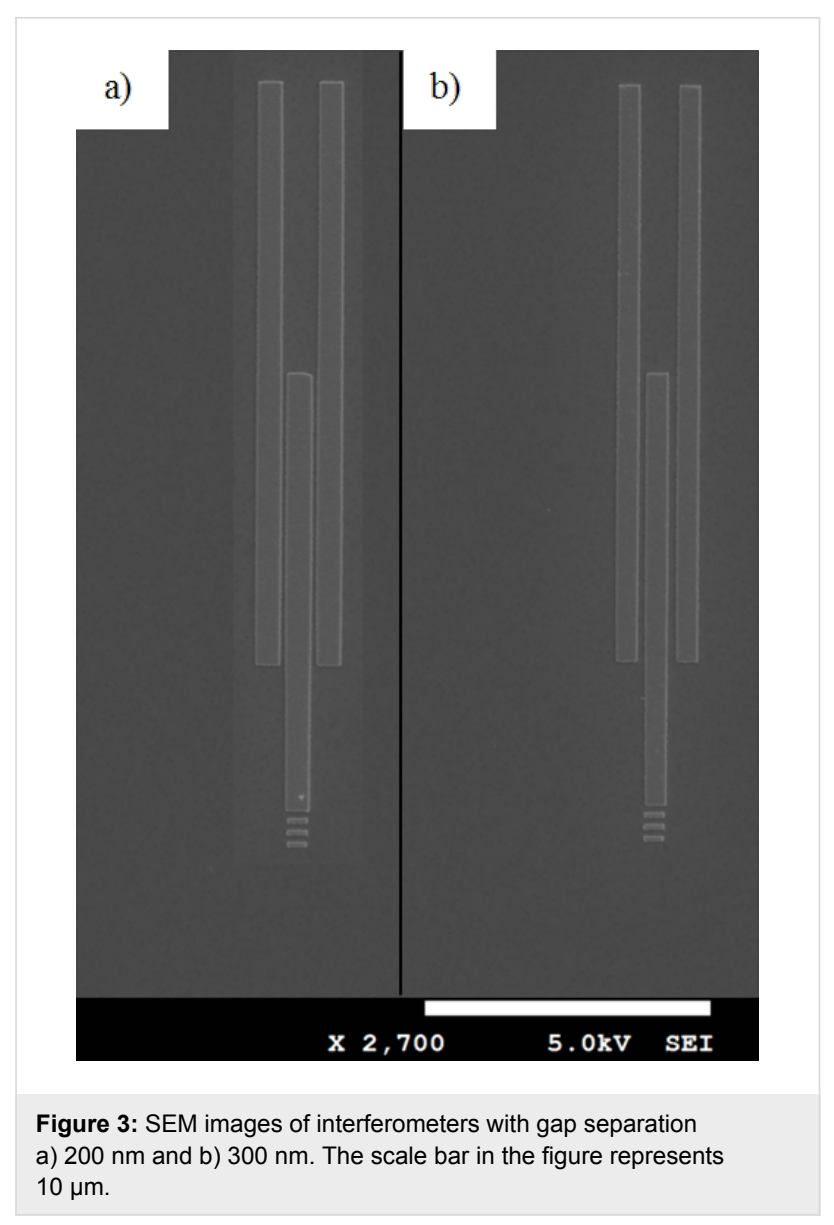

We observed that plasmon outcoupling intensity was higher when the outer waveguide separation is lower. Based on the experimental results obtained from the QD images we chose to proceed with the sensor with $200 \mathrm{~nm}$ outer arm separation.

An input laser of wavelength $633 \mathrm{~nm}$ and power level of $4.0 \mathrm{~mW}$ was used to excite the LRSPP mode in the input arm. The excitation setup is described in detail in [22]. A series of CCD images of the RI sensor in operation for different solutions with different refractive indices are shown in Figure 5. The CCD camera used was a DS-Ril with a sensitivity equivalent to ISO 200. The refractive index of the sample in the sample arm was varied by varying the sucrose concentration in deionized (DI) water, and the refractive index of the solution calculated using the details in [24].

Maximum incoupling was ensured by adjusting the beam position on the input grating such that it obtains maximum intensity. Incoupling and outcoupling intensities of the sensor were analysed using CCD images. The outcoupling intensity of the reference and sample arm were measured for each sucrose solution. Figure 6 depicts the relative intensity change $\Delta I / I_{0}$ between the sample and reference arm versus sucrose weight per- 


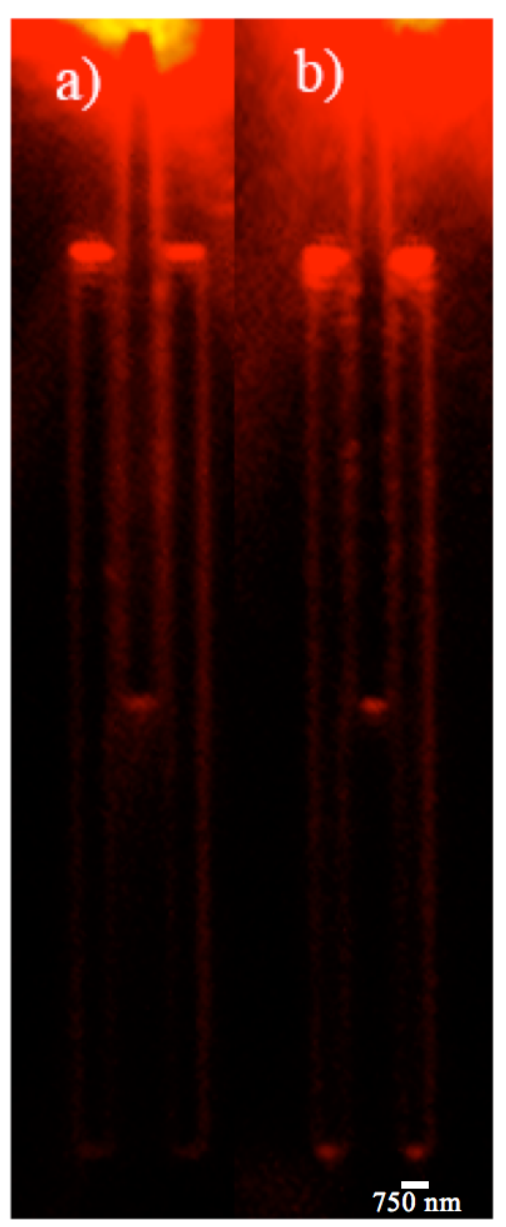

Figure 4: QD luminescence images of interferometers with $300 \mathrm{~nm}$ gap and $200 \mathrm{~nm}$ gap when excited via input arm with a $633 \mathrm{~nm}$ laser.

centage in each sucrose solution, where $I_{0}$ is the intensity on the reference arm, $I_{\mathrm{r}}$ (Equation 3).

$$
\frac{\Delta I}{I_{0}}=\frac{I_{\mathrm{r}}-I_{\mathrm{s}}}{I_{\mathrm{r}}} \times 100 \%
$$

$I_{\mathrm{S}}$ is the sample arm output intensity at a specific sucrose concentration (Equation 5). $I_{0}=I_{\mathrm{r}}$ is the reference arm output intensity and the reference arm was covered by PMMA of refractive index 1.4888 (at $633 \mathrm{~nm}, 20^{\circ} \mathrm{C}$ ) [24]. Five RI sensors are used to calculate the relative intensity change for each sucrose measurements (using Equation 6), and the results averaged. The error bars in Figure 6 are for the standard error in this averaging.

The relative intensity difference was highest for DI water (with no sucrose) and lowest for $10 \%$ sucrose in DI water. For further clarification, a graph was plotted against relative intensity change as a function of refractive index change with respect to PMMA (Figure 7). All the refractive indices of all sucrose solu-

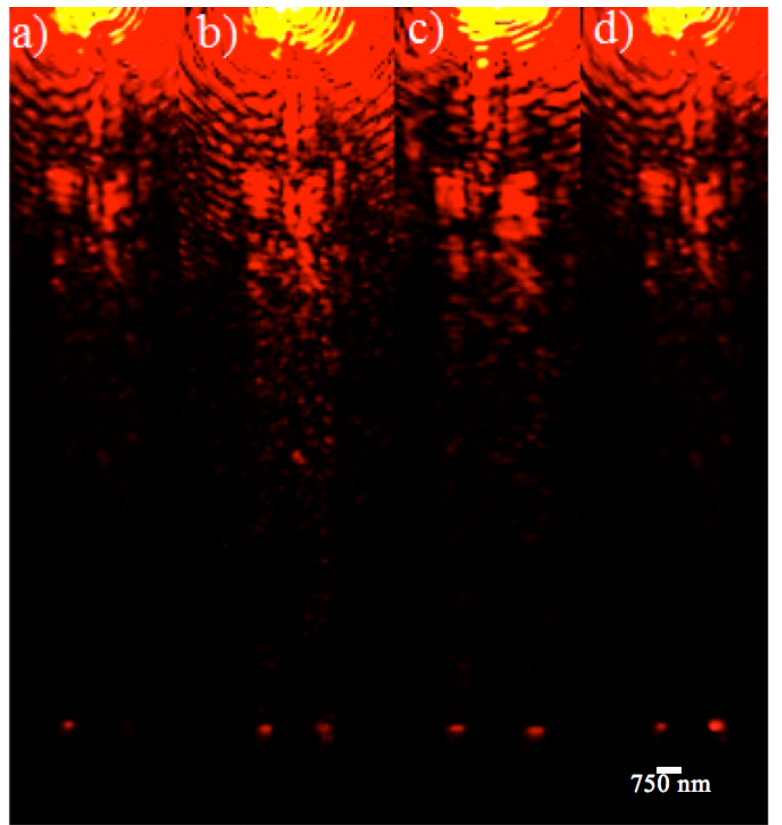

Figure 5: Plasmonic RI sensor. Right outer arm (reference arm covered with PMMA), left outer arm (sample arm - covered with $0.5 \%$ sucrose in DI water). Rightmost to left are series of CCD images obtained for $0 \%, 10 \%, 80 \%$ sucrose in DI water and index matching oil.

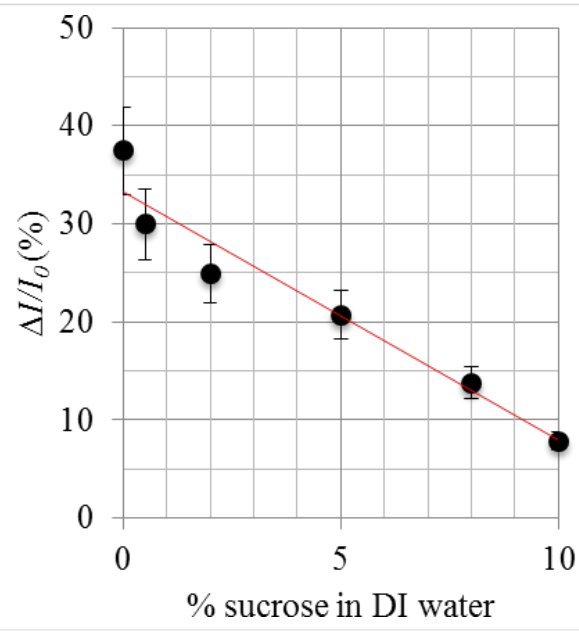

Figure 6: Relative intensity difference between the sample arm and reference arm versus weight percentage of sucrose in DI water. The error bars are for the standard error.

tions were measured at $20{ }^{\circ} \mathrm{C}$ at a wavelength of $633 \mathrm{~nm}$ relative to air [24].

We observed that the intensity difference between arms changes linearly with the change in the refractive index of the solution (w.r.t. PMMA). Figure of Merit (FOM) relative to the intensity change was calculated by dividing the difference in the relative intensity change $\Delta I / I_{0}$ by $\Delta n$. 


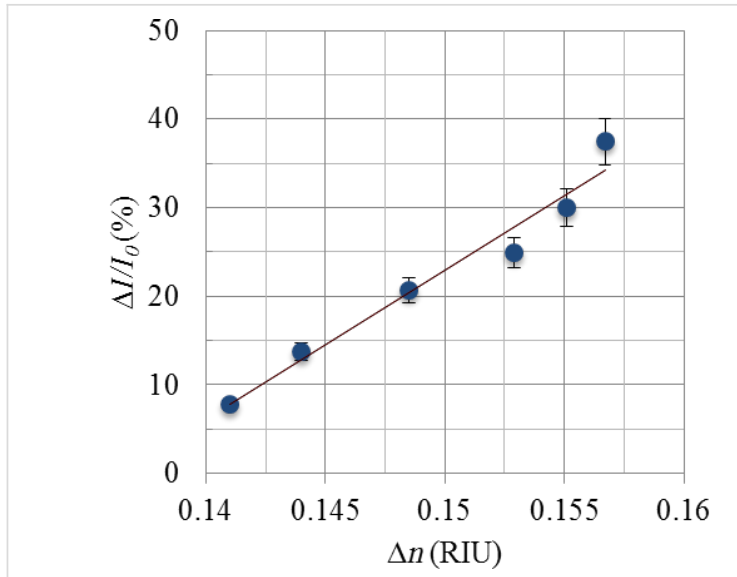

Figure 7: Relative intensity difference between sample arm and reference arm vs refractive index difference (between PMMA and sucrose solutions).

$$
\mathrm{FOM}=\lim _{\Delta n \rightarrow 0} \frac{\Delta I / I_{0}}{\Delta n}
$$

The calculated FOM for the relative intensity change of the device was $\approx 10^{3} \% /$ RIU. The gradient of the graph $\Delta I / I_{0}$ vs $\Delta n$ depicts the relative intensity difference for a refractive index change of 1 RIU in the sample arm. The device has a resolution of $6 \times 10^{-4}$ RIU for a change in relative output intensity of $1 \%$.

The sensor was further tested for $80 \%$ sucrose (R.I. 1.4906) and an index matching oil of RI 1.5150 . For $80 \%$ sucrose solution and for index matching oil, the sample arm intensity was higher than the reference arm (refer to CCD images on Figure $5 \mathrm{c}$ and Figure 5d).

\section{Conclusion}

We experimentally realised a three arm refractive index sensor design with a resolution of $\approx 6 \times 10^{-4}$ RIU for a $1 \%$ relative intensity change between output arms. The device is highly compact and authors believe it's the first attempt to fabricate an RI sensor with stripe waveguide coupling system.

\section{Supporting Information}

\section{Supporting Information File 1}

More details for the total field of the coupled system.

[http://www.beilstein-journals.org/bjnano/content/

supplementary/2190-4286-7-66-S1.pdf]

\section{Acknowledgements}

This work was performed in part at the Queensland node of the Australian National Fabrication Facility (ANFF), a company established under the National Collaborative Research Infra- structure Strategy to provide nano and micro-fabrication facilities for Australia's researchers. CP acknowledges valuable support from the UQ AIBN, UQ CMM, and QUT CARF team. $\mathrm{CP}$ and KV also acknowledge the QUT High Performance Computer facility. KV and EJ would also like to acknowledge the funding support of the following agencies - Australian Research Council (ARC) (DP110101454) and Asian Office of Aerospace Research and Development (FA2386-14-1-4056).

\section{References}

1. Zetie, K. P.; Adams, S. F.; Tocknell, R. M. Phys. Educ. 2000, 35, 46. doi:10.1088/0031-9120/35/1/308

2. Jacek, G.; Laurent, M.; Alain, D.; Sergey, I. B. Nanotechnology 2012, 23, 444008. doi:10.1088/0957-4484/23/44/444008

3. Gao, Y.; Gan, Q.; Xin, Z.; Cheng, X.; Bartoli, F. J. ACS Nano 2011, 5, 9836-9844. doi:10.1021/nn2034204

4. Zeng, X.; Gao, Y.; Hu, H.; Ji, D.; Gan, Q.; Bartoli, F. J. Appl. Phys. 2013, 113, 133102. doi:10.1063/1.4798942

5. Feng, J.; Siu, V. S.; Roelke, A.; Mehta, V.; Rhieu, S. Y.; Palmore, G. T. R.; Pacifici, D. Nano Lett. 2012, 12, 602-609. doi:10.1021/nl203325s

6. Lal, S.; Grady, N. K.; Kundu, J.; Levin, C. S.; Lassiter, J. B.; Halas, N. J. Chem. Soc. Rev. 2008, 37, 898-911. doi:10.1039/b705969h

7. Jackson, J. B.; Halas, N. J. Proc. Natl. Acad. Sci. U. S. A. 2004, 101, 17930-17935. doi:10.1073/pnas.0408319102

8. Nie, S.; Emory, S. R. Science 1997, 275, 1102-1106. doi:10.1126/science.275.5303.1102

9. Scholes, F. H.; Davis, T. J.; Vernon, K. C.; Lau, D.; Furman, S. A.; Glenn, A. M. J. Raman Spectrosc. 2012, 43, 196-201. doi:10.1002/jrs.3034

10. Bozhevolnyi, S. I.; Volkov, V. S.; Devaux, E.; Laluet, J.-Y.; Ebbesen, T. W. Nature 2006, 440, 508-511. doi:10.1038/nature04594

11. Liedberg, B.; Nylander, C.; Lunström, I. Sens. Actuators 1983, 4 , 299-304. doi:10.1016/0250-6874(83)85036-7

12. Homola, J.; Yee, S. S.; Gauglitz, G. Sens. Actuators, B 1999, 54, 3-15. doi:10.1016/S0925-4005(98)00321-9

13. Mehan, N.; Gupta, V.; Sreenivas, K.; Mansingh, A. Indian J. Pure Appl. Phys. 2005, 43, 854-858.

14. Feltis, B. N.; Sexton, B. A.; Glenn, F. L.; Best, M. J.; Wilkins, M.; Davis, T. J. Biosens. Bioelectron. 2008, 23, 1131-1136. doi:10.1016/j.bios.2007.11.005

15. Wu, X.; Zhang, J.; Chen, J.; Zhao, C.; Gong, Q. Opt. Lett. 2009, 34, 392-394. doi:10.1364/OL.34.000392

16. Fan, H.; Charbonneau, R.; Berini, P. Opt. Express 2014, 22, 4006-4020. doi:10.1364/OE.22.004006

17. Pezzé, L.; Smerzi, A. Phys. Rev. A 2006, 73, 011801. doi:10.1103/PhysRevA.73.011801

18. Gan, Q.; Gao, Y.; Bartoli, F. J. Opt. Express 2009, 17, 20747-20755. doi:10.1364/OE.17.020747

19. Vernon, K. C.; Gómez, D. E.; Davis, T. J. J. Appl. Phys. 2009, 106, 104306. doi:10.1063/1.3262625

20. Vernon, K. C.; Gomez, D. E.; Davis, T. J.; Tischler, N. J. Appl. Phys. 2011, 110, 074315. doi:10.1063/1.3650900

21. Berini, P.; Lahoud, N.; Charbonneau, R. J. Vac. Sci. Technol., A 2008, 26, 1383-1391. doi:10.1116/1.2975196 
22. Perera, C. S.; Vernon, K. C.; Funston, A. M.; Cheng, H.; Eftekhari, F.; Davis, T. J. Opt. Express 2015, 23, 10188-10197.

doi:10.1364/OE.23.010188

23. Perera, C. S.; Funston, A. M.; Cheng, H.-H.; Vernon, K. C. Beilstein J. Nanotechnol. 2015, 6, 2046-2051.

doi:10.3762/bjnano.6.208

24. Lide, D. R. CRC Handbook of chemistry and Physics; CRC Press: Boca Raton, FL, U.S.A., 2004; p 85.

\section{License and Terms}

This is an Open Access article under the terms of the Creative Commons Attribution License

(http://creativecommons.org/licenses/by/2.0), which permits unrestricted use, distribution, and reproduction in any medium, provided the original work is properly cited.

The license is subject to the Beilstein Journal of

Nanotechnology terms and conditions:

(http://www.beilstein-journals.org/bjnano)

The definitive version of this article is the electronic one which can be found at: doi:10.3762/bjnano.7.66 